\title{
Perception of Risks in Nanotechnology: Determining Key Aspects in Chile
}

\author{
J. POZO \\ Instituto de Ciencias Básicas. Universidad Diego Portales. Ejército 441 \\ Santiago, Chile. \\ E-mail: julio.pozo@udp.cl \\ www.udp.cl \\ A. SCHMESSANE \\ Instituto de Ciencias Básicas. Universidad Diego Portales. Ejército 441 \\ Santiago, Chile. \\ E-mail:ann_schme@yahoo.es \\ www.udp.cl \\ A. POZO \\ Universidad Diego Portales \\ Santiago, Chile. \\ E-mail: alejandra_pozo@yahoo.com \\ C. POZO \\ Universidad Diego Portales \\ Santiago, Chile. \\ E-mail: caritop80@yahoo.com \\ Received 18 April 2011; accepted 12 March 2012 \\ Abstract
}

\begin{abstract}
The purpose of this study is to provide preliminary base-line data on public perceptions about the risks associated with nanotechnology. We analyzed the current development of nanotechnology in Chile and the risks associated with the incorporation of this new technology over time, considering the particular case of Chile and its position in science and technology. A survey was applied to determine perceptions about the most prominent risks.
\end{abstract}

Keywords: Science, Nanotechnology, Nanomaterials, Technology, Legislation, Risks

\section{Introduction}

The size of nanoparticles represents one of the risks associated with nanotechnology, considering that the smaller a particle is, the greater its reactivity. Thus, we can infer that a particle that is inert at the micro or macro scale may be harmful at the nano scale. The possible risks associated with nanotechnology include nanoparticles penetrating the skin and entering the bloodstream and the interaction and effect of nanoparticles on eco- systems. Finally, there are economic effects associated with the proliferation of products in the market. ${ }^{1,2}$

Nanoparticles in contact with living tissue can cause the appearance of free radicals and cancer. Some studies have dealt with the potentially harmful consequences of nanoparticles to living beings and their rise through the food chain. ${ }^{3,4}$ Currently, scientific research and technological problems related to nanotechnology deserve great interest from both theoretical and experimental 
points of view. This is because the results that are being generated by describing their behavior try to solve many problems currently facing humanity, which to date have remained unsolved.

The objective of this study is to generate preliminary base-line information on public perceptions about the risks associated with nanotechnology.

\subsection{Theoretical analysis}

All changes in technology have associated economic changes. From the point of view of the economic importance of nanotechnology, NASA and the National Science Foundation have considered that the development of nanotechnology is of strategic importance. In the view of many people and institutions around the world, nanotechnology is producing a new industrial revolution. The financial and economic risks that NASA wants to avoid are evidenced by the fact it did not disclose the results of around 150 patent applications in the field of nanotechnology. ${ }^{5}$ Some first world economies have made significant investments in research, development and innovation (R \& D \& I) in nanotechnology. Therefore, it is expected that nanotechnology will have strong implications in future global economic trends, which may impact on almost all activities of our society.

\subsubsection{Nanotechnology, economics and global competitiveness}

It has been noted that in the very near future, the development of nanotechnology will revolutionize science, technology and human society. Nanotechnology has the potential to transform medicine, biotechnology, agriculture, manufacturing, material sciences, information technology, and telecommunications, among other fields.

The world is entering an era driven by accelerated technology projects and business ventures that generate significant economic value. We are in the middle of great changes in economic systems led by the rapid exponential growth of new technologies. Nanotechnology should be understood in this context, as such, it is a continuation in the acceleration of advanced technology and perhaps most importantly, it can have the capacity to transform the future global economy.
Ten years ago, America appeared to be the only country concerned with developing nanotechnology. Today, several countries have joined in this pursuit, such as China, India, Japan, Australia, Israel, Korea, and most recently Russia. All of them are becoming aware of the potential advantages of "betting" on this sector, while Europe seems to be more cautious. ${ }^{6}$

Significant advances in the field of nanotechnology and nanoscience have been achieved in the last two years, to the point that no one doubts that this field is set to become the ultimate strategic sector in the most advanced economies worldwide.

The evolution and growth of technology has become a cornerstone for the promotion of competitive innovation, the emergence of new businesses and prospects of economic progress for systems that strive to be players in the era of globalization and knowledge. While nanotechnological advances take place, economists and specialists are looking ahead to identify the impacts of the evolution and development of current technology both in economics and in business. Nanotechnology is critical because it can provide the necessary tools to facilitate a reorganization of society, business and the structure of our economies. 5

\subsubsection{Nanotechnology as a strategic sector in the international arena}

Nanotechnology projects can be divided into three phas$\mathrm{es}^{6}$. Nanotechnology is currently at the stage of research and the development of scientific knowledge, and is only beginning to have applications. During the next five years, many applications that are in the beginning stages are expected to be further developed. In the following ten years, nanotechnology will be consolidated as an industry, and consumers will be enjoying a wide range of products using nanotechnology.

\subsection{Overview}

The mechanical construction of molecules was first suggested by Richard Feynman, who is now considered the founder of nanotechnology. In an after-dinner speech entitled “There's Plenty of Room at the Bottom" at the California Institute of Technology in 1959, and later published in $1960^{7}$, Feynman proposed an alternative approach to miniaturization that would use ma- 
chines to build smaller machines, which could then build still smaller machines, and so forth. According to Feynman "The principle of physics, as far as I can see, do not speak against the possibility of maneuvering things atom by atoms. It is not an attempt to violate any laws; it is something, in principle, that can be done; but, in practice, it has not been done because we are too big”.

The prefix nano refers to one-billionth of something, for example a longitude of 1 nanometer $\left(1 \mathrm{~nm}=10^{(-9)} \mathrm{m}\right)$. The word nanotechnology is used extensively in relation to science and technologies at the nanoscale, an extremely small scale at which molecular structures and their atoms are manipulated, with the possibility of making building-materials and machinery from atomic and molecular arrays.

Currently, there are super microscopes that can move individual atoms using a probe approach, such as the scanning tunneling microscopy (STM), which is capable of creating moving images using electrically conductive needles on the atoms in a conductive surface. The STM was invented by Binnig and Rohrer at the IBM laboratory in Switzerland in 1982. In 1986, the inventors were awarded the Nobel Prize, a clear confirmation of the importance of STM. The STM maps topography by measuring the tunnel current between an atomically sharp tip and a metallic sample.

Another breakthrough came in 1986 with the invention of the atomic force microscope (AFM) by Binnig, Quate and Gerber. The AFM measures the force between a tip and a sample. In contrast to the STM, insulated samples can also be imaged. The AFM is suitable for different types of materials. The technique presented by these microscopes is very versatile and of great interest in the characterization of devices in the field of nanoscience and nanotechnology. In this context it has been possible to characterize the morphology of nonporous surfaces grown by electrodeposition, mechanical properties of metal surfaces in addition to significant magnetic properties.

\subsubsection{Definition of Nanotechnology}

Nanotechnology systematically organizes and manipulates properties and behaviors of matter at the atomic and molecular levels in order to create functional devic- es, materials and systems on a nanometer scale. In this context, the most frequently asked question is: What is Nanotechnology? The US National Science and Technology Council defines nanotechnology as: "The essence of nanotechnology is the ability to work at the molecular level, atom by atom, to create large structures with fundamentally new molecular organization. The aim is to exploit these properties by gaining control of structures and devices at atomic, molecular, and supramolecular levels and to learn to efficiently manufacture and use these devices”.

\subsubsection{Nanomaterials}

Nanomaterials are single or multi-phase polycrystalline structures with a typical size of 1 to 100 nanometers $(\mathrm{nm})$ in at least one dimension. The focus of nanomaterials is a bottom-up approach to structures and functional effects so that the building blocks of materials are designed and assembled in a controlled manner. Some examples are nanoclays that reinforce plastics or carbon nanotubes to add conductivity to various materials such as zinc oxide and cerium oxide. Zinc oxide is ideal for use in cosmetics and industrial application of radiation absorbing transparent ultraviolet light (UV), and antibacterial functions. Cerium is ideal for use in catalytic engines fuel cells and transparent UV absorbers.

In these nanomaterials, most atoms are located on the surface of the particles, augmenting or enhancing chemical, mechanical, optical and magnetic properties. Applications of nanomaterials include the generation of computer chips, phosphors for high-definition TV, highpower magnets, high-sensitivity sensors, more durable satellites, more durable medical implants and others. ${ }^{8}$

\subsubsection{Carbon Nanotubes}

Computer companies with large laboratories, such as IBM or HP, have research programs in nanotechnology. The interest is to obtain smaller electronic chips, so that when silicon technology reaches its limit, it will probably be replaced by a new concept which is called carbon nanotubes.

Nanotubes are composed entirely of carbon materials where the basic unit is a plane of graphite rolled into a cylinder, forming tubes whose diameter is on the order 
of a few nanometers. These single-walled tubes have properties that are stronger than steel.

\subsubsection{The Graphene}

This is a sheet of carbon one atom thick, which was first made in a laboratory in 2004. Graphite, which is the material commonly used in pencils, is made up of many layers of graphene. It should be noted that the conductive properties of graphene are highly efficient and can be used in nanoelectronics. In the form of a long ribbon of dimensions on the order of nanometers, graphene has unique electrical properties and behaves as a metal or semiconductor.

Graphene has a structure in which carbon atoms in flat sheets are joined, forming a hexagonal honeycomb, with one atom at each vertex. Graphite is obtained by placing many sheets on top of each other. Then if a portion of one of these films is wound in the form of a sphere, like a football, fullerenes are obtained (molecules of recent interest). If the structure related to the fullerenes is rolled into a cylinder, it will form a carbon nanotube. Within this field, a graphene honeycomb corresponds to a single extended two dimensional flat structure.

\subsubsection{Dry Nanotechnology}

Dry nanotechnology deals with the manufacture of structures in carbon (carbon nanotubes), silicon, inorganic materials, metals and semiconductors, and is used in electronics, magnetism and various optical devices, in addition to being controlled by selfassembled computers.

\subsubsection{Wet Nanotechnology}

This technology is based on biological systems in wet environments, considering genetic material, membranes, enzymes and other cellular components. In brief, we can say that wet nanotechnology is used in the context of biological cell division and DNA.

\section{Frame of Reference of Nanotechnology}

The frame of reference of nanotechnology is understood as the context in which it develops and is implemented. This context is composed of interest groups. The interest group is understood as all those companies or people that participate in processes associated with the development of this technology. These participants can be classified into 6 groups: The state, the society, consumers, universities, engineers and scientists, and businesses. All these groups can express their opinions regarding this technology, besides developing it or simply being affected by the benefits or risks of nanotechnology. Particularly, we should understand that this tool functions and operates in a context and the possible risks or benefits depend on the use that these groups give to it.

The role of the interest groups in the development of nanotechnology: The State and businesses. They have the obligation to regulate and control the use of nanotechnology, as well as rewarding the good use, since this encourages the adequate utilization of this technology. Engineers, scientists and universities should educate, conduct outreach, and invest in nanotechnology to develop awareness in the population and among students. The society and consumers are the recipients of this technology. The contribution of these groups is not only to observe the evolution of and enjoy the possible benefits that this technology, but also to be informed and express their opinions on these themes. ${ }^{9,10}$

\subsection{Implementation process of nanotechnology}

The implementation of nanotechnology is composed of 3 stages:

The initial phase that aims to evaluate the actual versus the expected, in order to analyze the differences between what is and what is desired in nanotechnology.

The second stage is to coordinate planning and the possible mass evolution of this technology in several years. The development stage involves all the processes to implement nanotechnology. These include regulation and legislation, investment strategies.

The third and final stage is related to results because it is here where we can see and measure whether the technological benefits outweigh the risks. 


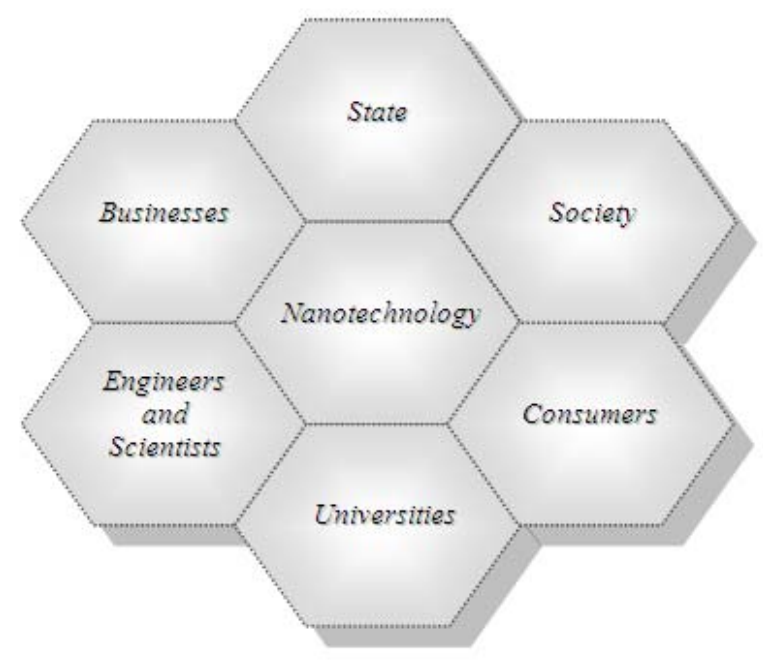

Fig. 1. Schematic diagram of the interest groups that make up the frame of reference for nanotechnology.

\section{Responsible Nanoscience and its Meaning}

Some background: In 2008, The European Commission recommended a code of conduct for responsible nanosciences and nanotechnologies research. This is one of a growing number of codes of conduct being proposed for nanotechnology. In the case of the European Union (EU), the main goals are commendable. It seeks a relationship among everyone involved in academic research and industry. The UK government recently issued the Universal Ethical Code for Scientists, which considered the following general principle "ensure that your work is lawful and justified", and one injunction to "minimize and justify any adverse effect your work may have on people, animals and the natural environment". ${ }^{11}$

\subsection{Responsible Nanotechnology (RN)}

Responsible Nanotechnology is a relatively new concept that refers to a responsible management of potential risks, and the promotion of the benefits on behalf of the human kind. The potential of the nanotechnology from a social point of view is immense, as are the potential risks due to irresponsible use or management. The academic community that defends the concept of responsible nanotechnology pursues a vision of the world in which molecular production is utilized for beneficial and productive purposes, and in which evil uses of their potential is prevented by an efficient management of the technology. In the United States, the Center for Responsible Nanotechnology (CRN) promotes debate related to the responsible management of nanotechnology. To offer complete and precise information, clear explanations and feasible proposals, CRN studies all aspects of nanotechnology and its impact. This includes technological, social, military, economic, and political sides. This center presents the results of its studies to specialized public, but also to the general public, and tries to present the information in the most effective way possible. The objective of CRN is to investigate the ethical, legal and social implications of molecular production and to educate the people so they influence its development and applications. $^{12}$

\section{Some Risks Associated with Nanotechnology}

It is important to note that in October 2004 the UK Health and Safety Executive estimated that more than 10,000 workers dealing with nanoparticles could be exposed to some risks, in addition to not using effective methods of protection to avoid ingestion and inhalation of or dermal exposure to nanoparticles in the production process.

In 2005, the U.S. National Institute of Occupational Safety and Health reported that significant damage was found in the DNA of the heart and arteries of mice exposed to carbon nanotubes. In the same year, a NASA study reported that the injection of commercially available carbon nanotubes (equivalent to 17 days of exposure for a worker) caused significant damage in the lungs of rats. Researchers at the University of Rochester reported that rabbits subjected to inhalation of carbon nanospheres showed increased susceptibility to blood clots. At the meeting of the American Chemical Association, a report ${ }^{13}$ was presented that showed that carbon nanoparticles dissolve in water and that even very small concentrations are toxic to soil bacteria. This caused alarm about possible interactions with natural ecosystems.

In 2003, a study published in Nature ${ }^{14}$ showed that nanoparticles can be absorbed by earthworms and other soil organisms, with the possibility of moving up the food chain. Therefore, there is concern about the risks of nanoparticles in humans, but these risks will only be known over time. To date no real impact is known, oth- 
er than several studies that suggest certain dangers. These risks are perhaps no greater than those of existing industries $^{15 .}$

\subsection{Risk Factors}

In the context of the Responsible Nanotechnology (RN) and to facilitate its comprehension as a model to minimize the risks associated with nanotechnology in Chile $^{16}$, we developed a model that could be helpful for other countries as well. A mathematical relationship that incorporates the most prominent factors to consider is proposed in this work. This relationship takes into consideration the following fundamental aspects:

- Legislation (L)

- $\quad$ Funding for Research (FR)

- $\quad$ Sanctions for Undue Use (SUU)

- $\quad$ State Control (SC)

- $\quad$ Good Practice Rewards (GPR)

As such, the model is represented by the following equation ${ }^{17}$ :

$$
\begin{aligned}
R N= & L\left(w_{L}\right)+S R\left(w_{S R}\right)+P U U\left(w_{P U U}\right) \\
& +S C\left(w_{S C}\right)+G P R\left(w_{G P R}\right)
\end{aligned}
$$

Where: $w_{\mathrm{L}}, w_{\mathrm{SR}}, w_{\mathrm{SUU}}, w_{\mathrm{SC}}$ and $w_{\mathrm{GPR}}$ are their respective weights.

Why do we consider these aspects and not others? The answer is simple, because investment in this field in Chile by the private sector is low. Thus, public funding of research is very important.

Technology in Chile is based principally on imports, so state control is important. The question is the following: How to control the revenues for safe technologies in the country? We think the above can be obtained by differential taxes and funding.

\section{Nanotechnology in Chile}

To contextualize the situation of nanotechnology in Chile, it is important to consider current legislation or regulations as a reference framework, that is to say, a methodology that describes and discriminates between the correct and the incorrect. It would be desirable that regulation for the use of the nanotechnology is as detailed as possible, without leaving room for different interpretations, or in other words, that it has a clear meaning that allows for common understanding.

Government funding will also be important because it will allow for the development of new topics in research and development and innovation (R\&D\&I) in nanotechnology, which is lacking in Chile and is of an immediate need. $^{18}$

Chile must identify nanotechnology as a strategic sector of development, and must consider a number of initiatives in order to maximize profits, while minimizing their risk.

Others studies ${ }^{19,20}$ have shown the importance of public perceptions for the successful development and implementation of new technologies. Perceptions can modify the direction, and behavior of scientific activity in many fields of research and technologic development. A relevant example is nuclear energy.

To identify public concerns regarding nanotechnology, we conducted a survey to detect perceptions and knowledge about the risks and benefits of nanotechnology. This survey included questions about its regulation, and the possible impact and consequences of this technology in Chile.

Because there are no previous reports regarding Chilean public opinion on this matter, our first approximations were based on surveys directed to two discrete groups; the general public, and university students and professors (academic world).

The intention is to find differences between the two groups, because science and technology at the nanoscale is studied in Chile only in the academic world by research groups in few universities.

The issues contemplated in the survey are the ingredients for responsible nanotechnology in Chile, the proportion of them in the equation respond principally to the cultural framework in Chile and how Chilean society responds. 


\subsection{Chile as a competitive country in 2020}

Chileans should be aware of what is happening with new technologies to be better prepared for future challenges. With regard to nanotechnology, Chile was selected by the World Bank as pioneer by the Millennium Science Initiative, which has been funding some projects in nanotechnology.

\subsection{Human Resources in Chile and S \& $T$ Policies}

Human resources are one of the predominant factors in the scientific and technological development of a country. A poor educational policy or its delay may result in the loss of international competitiveness.

\section{Opinion Survey}

To evaluate the status of nanotechnology in Chile, we conducted an opinion survey. Detailed information on the specific questions and the complete results can be found $\mathrm{in}^{21}$. The survey was applied to different representative sectors of Chilean society represented by 200 people divided into two groups of 100 each. The first group included the general public (private and public sector), and the second consisted of university students and professors. The survey had 15 questions, grouped as follows: 4 questions related to perceptions about nanotechnology, 3 related to the respondent's knowledge of nanotechnology, 2 about personal opinions, 3 about risks associated with nanotechnology, and the last 3 about responsibility at the country level.

\subsection{Survey Results}

There was no difference between the two groups, which allowed us to emphasize the following aspects that are prominent $^{21}$ :

\section{- Perception}

Although the majority of respondents had heard about nanotechnology and respondents were interested in knowing more about $i^{21}$, they considered that the level of development and investments made in Chile in this field are low (Fig. 2).

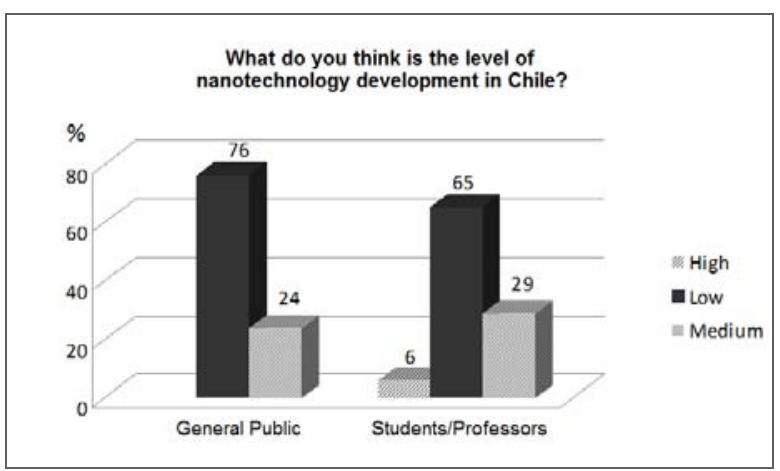

Fig. 2. A sequence of responses that involves percentages versus number of people for the two groups (general public and students/professors) in connection with the question.

\section{- Knowledge of its applications}

In relation to the applications of nanotechnology, close to $50 \%$ of respondents thought that the applications are in informatics, $24 \%$ in medicine, $20 \%$ in development of products and materials and 8\% in foods (Fig. 3).

Most of the respondents also thought that the level of knowledge about the theme depends more on motivation and personal interest than educational level and socioeconomic status. $^{21}$

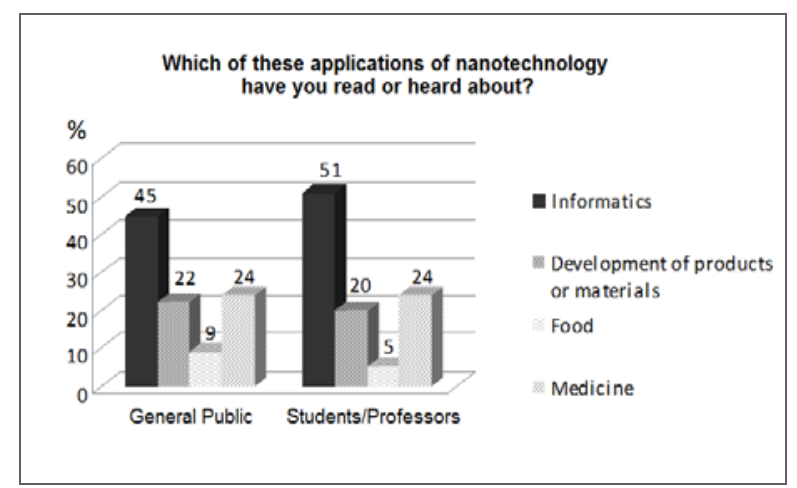

Fig. 3. A sequence of responses that involves percentages versus number of people for the two groups (general public and students/professors) in connection with the question.

\section{- Opinion}

Almost all respondents stated that no regulations exist in Chile (Fig. 4). 70\% stated that public opinion should be 
considered in developing nanotechnology in Chile (Fig. $5)$.

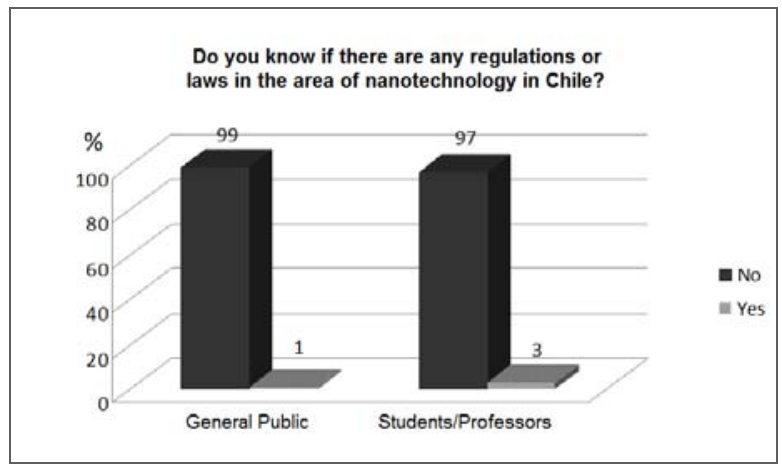

Fig. 4. A sequence of responses that involves percentages versus number of people for the two groups (general public and students/professors) in connection with the question.

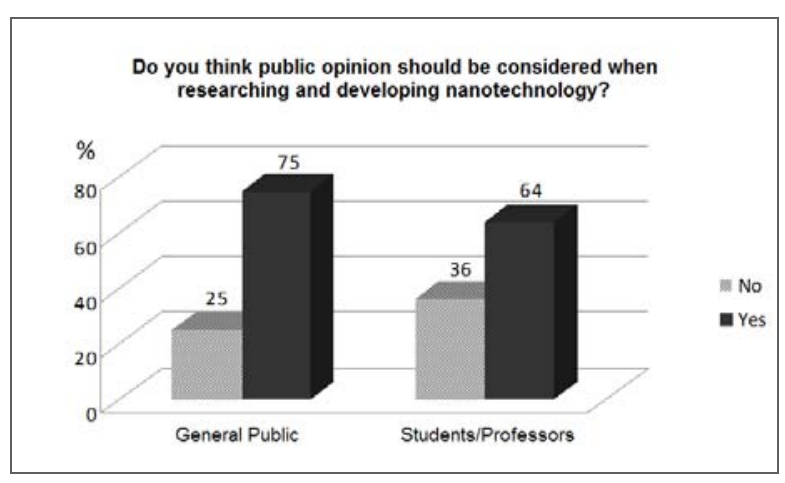

Fig. 5. A sequence of responses that involves percentages versus number of people for the two groups (general public and students/professors) in connection with the question.

\section{- Associated risks}

Regarding potential risks, 55\% thought that nanotechnology can harm both people's health and the environment, with about 30\% thinking that it can be harmful for peoples' health and 15\% concerned about environmental risks (Fig. 6). Nevertheless, 90\% of respondents in both sectors stated that they did not know what risks may be associated with handling nanomaterials (Fig. 7). Besides, more than $90 \%$ of respondents considered that nanotechnology may improve global competitiveness (Fig. 8).

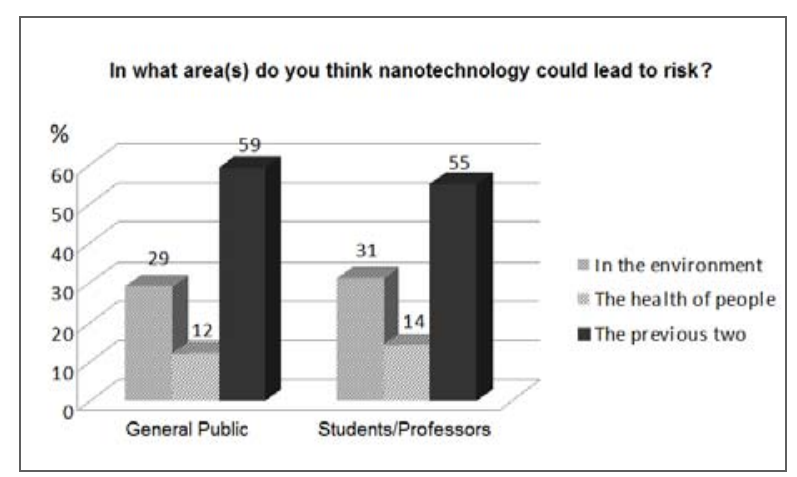

Fig. 6. A sequence of responses that involves percentages versus number of people for the two groups (general public and Students/professors) in connection with the question.

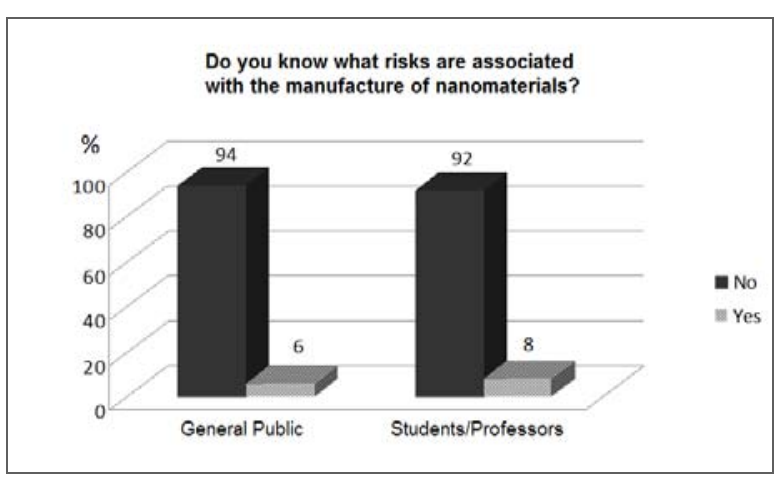

Fig. 7. A sequence of responses that involves percentages versus number of people for the two groups (general public and Students/professors) in connection with the question.

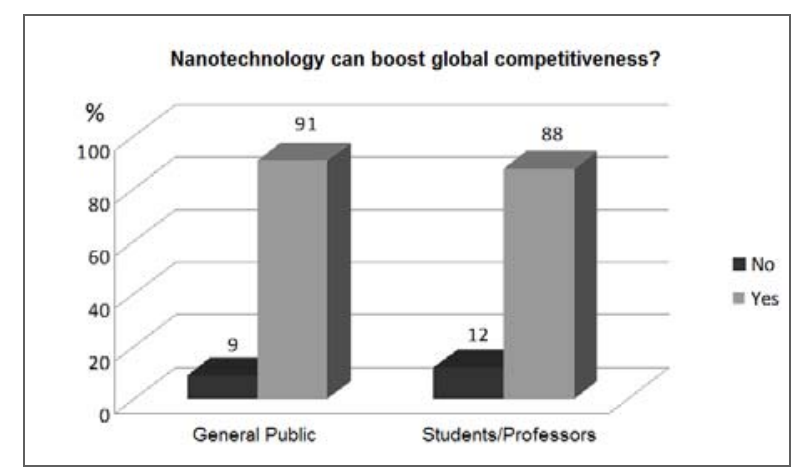

Fig. 8. A sequence of responses that involves percentages versus number of people for the two groups (general public and students/professors) in connection with the question. 


\section{- Responsibility at the country level}

On the other hand, close to $90 \%$ of respondents considered that the development of nanotechnology is a pending challenge for Chile (Fig. 9). Regarding who should finance research, $44 \%$ identified the private sector, $42 \%$ the State and 14\% universities (Fig. 10).

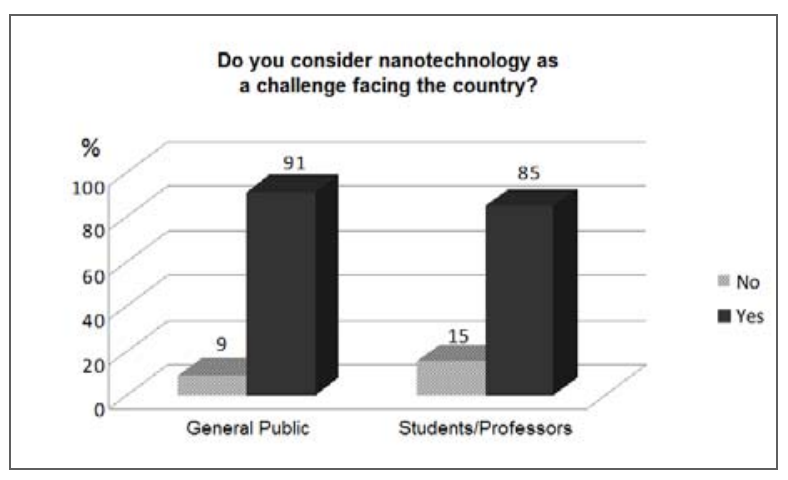

Fig. 9. A sequence of responses that involves percentages versus number of people for the two groups (general public and students/professors) in connection with the question.

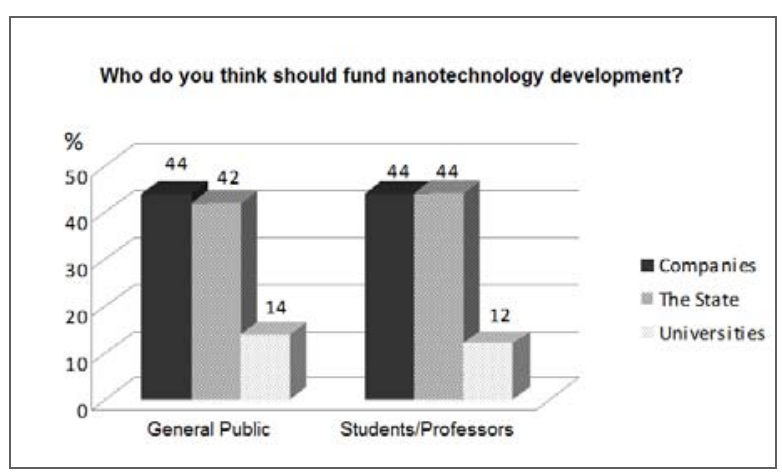

Fig. 10. A sequence of responses that involves percentages versus number of people for the two groups (general public and students/professors) in connection with the question.

\section{Conclusions}

The two groups (general public, and university students and professors) tended to give similar answers to all the questions. It is clear that the discussion in Chile on nanotechnology is incipient and that the same level of information is available to everyone. Based on this sur- vey, it should be the responsibility of the Chilean government to propose legislation in science and related technology in the context of research and development and innovation (R\&D\&I) that establish the norms to regulate the development of nanotechnology in Chile. Funding for research in nanotechnology should be provided by both the government and large Chilean businesses.

Additionally, it is necessary to emphasize that the universities and institutes should be responsible to for developing adequately trained engineers and scientists with the ethical disposition to face the challenges of the future in this matter. ${ }^{5}$

Based on the information and the results generated in this work, and with the help of decision tree ${ }^{22}$, it is possible to assign relative weight factors (\%) to each parameter in the equation (1).

Given that Chile does not have legislation yet (L), and sanctions for the undue use (SUU) to start is also important, both parameters are weighted with $30 \%$ followed by funding for research (RF) with $20 \%$, State Control (SC) and Good Practice Rewards (GPR) both with $10 \%$ each. Thus, the suggested model is as follows:

$$
\begin{aligned}
R N= & L(30 \%)+S R(20 \%)+P U U(30 \%) \\
& +S C(10 \%)+G P R(10 \%)
\end{aligned}
$$

This equation may be useful to acquire preliminary quantifiable baseline information.

As an emerging field, the development of nanotechnology, by both the private and public sectors, should be undertaken with consideration of social responsibility. Another aspect can also be considered in our model as the self-regulation by the society and the responsibility of the private sector. Nevertheless the last aspect in our model GPR is zero, because the development of science and technology in Chile is low.

We think that any further development of this technology in Chile should involve adequate legislation.

We conclude that the main risks associated with nanotechnology relate to the lack of legislation and/or regulations in Chile in the area of science and technology, 
which results in a lack of leadership, and therefore a deficit in the construction of knowledgement in term of what are the determining factors in global economic dynamics. There are few specialists in Chile to address the issue. Finally, there should be a communication strategy to educate citizens, which is of vital importance to establish public policies and regulations on the subject.

\section{References}

1. M. Siegrist, C. Keller, H. Kastenholz, S.Frey and A. Wiek Laypople's and Experts' Perception of nanotechnology Hazards, (Risk Analysis, Vol. 27, No 1, pp. 59-69, 2007).

2. Theories and Applications of Risk Analysis and Crisis Response The First International Conference on Risk Analysis and Crisis Response, (September 2526, 2007, Shanghai, China). http://racr.shmtu.edu.cn/en_index.asp

3. Chi-Fai Chau, Shiuan-Huei Wu and Gow-Chin Yen The development of regulations for food nanotechnology (Trends in Food Science \& Technology 18, 269-280, 2007).

4. K. Thomas, P. Sayre Research strategies for safety evaluation of nanomaterials, parts I: evaluating the human health implications of exposure to nanoscale materials (Toxicological Science 87 (2) pp, 316-321, 2005).

5. J. Pozo, A. Schmessane, G. Estay Los Ingenieros chilenos la Nanotecnología y las estrategias económicas a futuro (Actas CD XXIV Congreso Chileno de Educación en Ingeniería, SOCHEDI 2010).

6. A. Pedreño Muñoz Nanotecnología y Economía: estrategias de futuro (Instituto de Economía Internacional. Universidad de Alicante. URL) http://iei.ua.es/nanotecnologia/nanotecnologia-yeconomia-estrategias-de-futuro

7. R. P. Feynman, (Engineering and Sciences Vol. 23, 22-36, 1960)

8. Azom.com Nanomaterials and Their Applications (Nov. 23, 2001). http://www.azom.com/article.aspx?ArticleID=106 6

9. Igor Linkov et. al. Emerging methods and tools for environmental risk assessment (Journal of Nanoparticle Research 11: 513-527, 2009).
10. Management Principles for Nanotechnology (Nanoethics 2:43-60, 2008).

11. R. Jones Are you a responsible nanoscientist? (Nature Nanotechnology 4, 336 2009)doi:10.1038/nnano.2009.127

12. Center for Responsible Nanotechnology 20012008 http://www.crnano.org/

13. A. Johansen et al. Effects of $C_{60}$ fullerene nanoparticles on soil bacteria and protozoans (Article first published online: 9 DEC 2009). DOI: 10.1897/07-375.1

14. G. Brumfiel, Nanotechnology: A little knowledge (Nature, Vol. 424 pp. 246-248, 17 July 2003).

15. D. Tomanek, The Nanotube Site 23 Nov. 2005. http://www.pa.msu.edu/cmp/csc/nanotube.html

16. J. Pozo and A. Schmessane Nanomaterials, the risks and benefit for the Society and the Environment (Proceedings I Latin American Congress SRA-LA 2010, page 33).

17. Parlamento Europeo, Comisión de de Industria Investigación y Energía. Sobre nanociencias y nanotecnologías: un plan de acción para Europa 2005-2009.

http://www.europarl.europa.eu/sides/getDoc.do?pu bRef=-//EP//TEXT+REPORT+A6-20060216+0+DOC+XML+V0//ES

18. M. C. Roco International perspective on government nanotechnology funding in 2005 (Journal of Nanoparticle Research, 7: pp. 707-712, 2005).

19. M. Cobb and J. Macoubre Public perceptions about nanotechnology: Risks, benefits and trust (Journal of Nanoparticle Research 6: 395-405, 2004).

20. S. Currall, E.King, N. Lane, J. Madera and S. Turner What drives public acceptance of nanotechnology (Nature Nanothecnology Vol I December 2006).

21. J. Pozo A. Schmessane, A. Pozo y C. Pozo. Nanotechnology Survey Chile 2011. http://nanotecnologiasurvey.blogspot.com/

22. H. Arsham Herramientas para el Análisis de Decisión: Análisis de Decisiones Riesgosas. http://home.ubalt.edu/ntsbarsh/opre640s/spanishp. htm 